\title{
Experiencia de trasplante renal en la práctica privada en el Hospital Ángeles del Carmen
}

\author{
Kidney transplant experience in private practice at Hospital Ángeles del Carmen
}

\author{
Gonzalo Agustín García y Otero, ${ }^{*}$ Claudia Alejandra Aceves Quintero, ${ }^{\ddagger}$ Juan Carlos Corona Meléndez
}

\section{Resumen}

Introducción: Existe poca información de trasplantes en la práctica privada en países en desarrollo. Se documentó la experiencia en trasplante renal en CENREN y el Hospital Ángeles del Carmen. Material y métodos: Se realizó un estudio de cohorte retrospectivo obteniendo variables demográficas, tiempo con nefropatía, terapia sustitutiva, analítica química, función renal actual y supervivencia. Resultados: Se trasplantaron 58 pacientes en el periodo 2005-2018; 43.4\% de donador vivo relacionado (DVR), $13.2 \%$ vivo no relacionado (DVNR) y $43.4 \%$ cadavérico (DC). La sobrevida a cinco años fue $88 \%$ en receptores de DC, $94 \%$ DVR y $100 \%$ DVNR. $13.8 \%$ presentaron rechazo crónico (26.1\% DC, $14.3 \%$ DVNR y $4.3 \%$ DVR, $p<0.05$ ). $20 \%$ de los receptores de donador cadavérico necesitaron TSR posterior al trasplante, $16.7 \%$ de vivo no relacionado y ninguno de vivo relacionado, siendo factor de riesgo de rechazo (RR 37.0, IC 95\% de 5.35-255.7, $\mathrm{p}<0.05$ ); $93.8 \%$ de donador vivo relacionado mantienen KDIGO G1-3 (83.3 y 84.6\% DVNR y DC, respectivamente). Conclusión: LoS resultados en la práctica privada en México son parecidos a los reportados en países desarrollados, algunos incluso mejores, como el tiempo de isquemia fría en trasplante cadavérico, uso de basiliximab y función renal a cinco años.

Palabras clave: Trasplante renal, medicina privada, México.

\section{INTRODUCCIÓN}

El primer trasplante renal exitoso en un ser vivo fue realizado en un perro por Emerich Ullmann en 1902. En 1909 se hicieron esfuerzos para trasplantar riñones de cadáver humano a monos, y en 1910 se llevó a cabo el primer xenotrasplante en

\section{Abstract}

Introduction: There is little information on transplantation in private medical practice, particularly in developing countries. We documented the renal transplant experience at CENREN and Hospital Ángeles del Carmen. Material and methods: A retrospective cohort study was conducted, obtaining demographic variables, time with nephropathy, renal replacement therapy (RRT), chemical analysis, renal function and survival. Results: 58 patients were transplanted between 2005-2018. 43.4\% were from a living related donor (LRD), 13.2\% living unrelated donor (LUD) and $43.4 \%$ cadaveric donor (CD). The 5-year survival was $88 \%$ in recipients from CD, $94 \%$ LRD, and $100 \%$ LUD. Chronic rejection occurred in $13.8 \%(26.1 \%$ CD, $14.3 \%$ LUD and $4.3 \%$ LRD, $p<0.05$ ). $20 \%$ of cadaveric donor recipients required posttransplant RRT, $16.7 \%$ from living unrelated donor and none from the living related did, considering this as a risk factor for rejection (RR 37.0, 95\% Cl 5.35-255.7, p < 0.05). 93.8\% of living related donor maintains a KDIGO G1-3 (83.3 and $84.6 \%$ in recipients of living unrelated and cadaveric, respectively). Conclusion: Results in kidney transplant in private medical practice in Mexico are similar to those reported by developed countries, some even better such as time of cold ischemia in cadaveric donor transplantation, use of basiliximab and renal function at five years.

Keywords: Kidney transplantation, private medicine, Mexico.

humanos con poco éxito, a pesar de los intentos con perros, monos, cabras y ovejas. En 1939 el ruso Yurii Voronoy realizó un intento fallido de trasplante de alogénico, mientras que Jean Hamburger lo consiguió en 1953 en París de donador vivo relacionado (a un joven de 16 años de su madre). En 1954 Murray realizó en Boston el primer trasplante exitoso

\footnotetext{
* Medicina Interna-Nefrología. Director médico del Centro de Tratamiento de Enfermedades Renales (CENREN).

₹ Médico pasante de Servicio Social en el Centro de Tratamiento de Enfermedades Renales (CENREN).

$\S$ Medicina interna. Jefe de la Unidad de Cuidados Intensivos del Hospital Ángeles del Carmen.

Correspondencia:

Dra. Claudia Alejandra Aceves Quintero

Correo electrónico: dra.acevesq@gmail.com

Aceptado: 21-11-2019.

www.medigraphic.com/actamedica

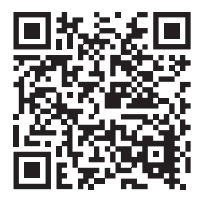


a largo plazo (ocho años) en gemelos monocigóticos, lo que le llevó a la obtención del Premio Nobel de Medicina en 1990. El primer trasplante de donador vivo no relacionado y de donador cadavérico se efectuó en 1962 con el surgimiento de fármacos inmunosupresores, obteniendo mejores resultados con azatioprina y esteroides. ${ }^{1-3}$ Desde entonces, el trasplante renal ha ganado cada vez más terreno en el tratamiento de la enfermedad renal crónica terminal (ERCT), hasta consolidarse como el tratamiento de elección definitivo. En Estados Unidos durante el periodo de 1988 a 2007 se reportaron 159,119 trasplantes de donador cadavérico y 83,471 de donantes vivos. ${ }^{4}$

El primer trasplante en Latinoamérica se realizó en la Ciudad de México en 1963 en el Centro Médico Nacional del Instituto Mexicano del Seguro Social y el primer trasplante renal en Guadalajara, Jalisco fue en $1976 . .^{5}$ En términos reales, en México ocurre un trasplante renal por cada 454 habitantes, mientras que en España se realiza un trasplante renal por cada 204 habitantes. Para hacer más evidente esta diferencia, en el periodo 1990-2009 se registraron en México 24,587 trasplantes de riñón, mientras que en el mismo periodo España reportó 39,798 procedimientos. ${ }^{6}$

Es de conocimiento general que en comparación con países desarrollados, los países en vías de desarrollo tienen tasas bajas de trasplante debido a una infraestructura menor, falta de personal entrenado, además de factores sociales, culturales y económicos. La falta de recursos en países en vías de desarrollo es un factor importante para la poca actividad de trasplantes realizados, con una tasa de 10 por cada millón de población, a diferencia de los países desarrollados con tasas de 45-50 por cada millón de población. 7,8

Monteón y colaboradores reportaron la experiencia más grande en trasplante renal en México, que abarca de agosto de 1976 a octubre de 2003 en el Centro Médico de Occidente del Instituto Mexicano del Seguro Social en Guadalajara, Jalisco. ${ }^{5}$ Durante este periodo se realizaron 1,356 trasplantes renales, 1,218 (90\%) de donador vivo (112 no relacionado) y 138 (10\%) de cadáver. MoralesBuenrostro y su equipo describieron la experiencia de 40 años de trasplante renal en el Instituto Nacional de Ciencias Médicas y Nutrición Salvador Subirán de la Ciudad de México, que incluyó 923 trasplantes. ${ }^{9}$ El análisis de la sobrevida del injerto a uno, cinco y 10 años resultó de 92, 83 y 74\%, respectivamente, con una media de 24.5 años. Asimismo, Contreras y colaboradores reportaron la experiencia en trasplante renal en el Hospital Ángeles Pedregal, la cual incluye 29 trasplantes renales realizados durante el periodo de enero de 2011 a diciembre de 2017, de los cuales $37.9 \%$ fueron de origen cadavérico y $62.1 \%$ de donador vivo. ${ }^{10}$

La República Mexicana es un país de contrastes en los rubros cultural, social, económico, educativo y en acceso a los servicios de salud. Desde zonas de alto grado de mar- ginación con la más rústica e improvisada infraestructura sanitaria, con poca posibilidad de trasplante y seguimiento de éste, hasta los sofisticados y especializados centros de medicina privada en las grandes ciudades que se equiparan en gran medida a los recursos disponibles en países desarrollados. La experiencia descrita en los estudios mexicanos previos proviene principalmente de hospitales públicos que atienden población con nivel socioeconómico bajo y medio, y sólo se encontró el reporte de un hospital privado que atiende población de nivel socioeconómico medio-alto y alto. Por lo tanto, queda de manifiesto la importancia de contribuir a la información de trasplantes en la práctica de la medicina privada en México.

La experiencia en trasplantes es de gran utilidad para conocer el comportamiento de la evolución de éstos en el tipo de población que maneja, así como su comparación con los resultados obtenidos tanto en países desarrollados como su contraparte en países en vías de desarrollo. El objetivo principal fue documentar la experiencia en trasplante renal en pacientes atendidos en el ámbito de la medicina privada por el centro de tratamiento de enfermedades renales (CENREN) y el Hospital Ángeles del Carmen, así como comparar las características demográficas, clínicas y bioquímicas de acuerdo con el origen del riñón trasplantado, la tasa de rechazo crónico de acuerdo con el origen del riñón trasplantado y la sobrevida y grado de función renal postrasplante.

\section{MATERIAL Y MÉTODOS}

Se realizó un estudio de cohorte retrospectivo desde 2005 hasta 2018, incluyendo todos los pacientes trasplantados en el Hospital Ángeles del Carmen mayores de 16 años, atendidos en consulta privada en CENREN y enviados para trasplante a dicho hospital. Se obtuvieron variables demográficas y relacionadas al trasplante del expediente del archivo clínico del hospital, y se complementó la información con el expediente de los pacientes de la consulta externa, tanto para información previa al trasplante así como para el seguimiento postrasplante. Se obtuvieron las variables de edad, género, antecedentes, tratamiento previo al trasplante, tiempo con enfermedad renal crónica previo al trasplante, tipo de terapia sustitutiva renal, laboratorios al ingreso hospitalario para trasplante, al egreso hospitalario del trasplante, y los más actuales durante el último año, necesidad de terapia sustitutiva renal con diálisis postrasplante, grado de función renal actual y supervivencia. Se realizó un análisis comparativo de las variables por origen del riñón trasplantado (donador vivo relacionado, vivo no relacionado o cadavérico) y de acuerdo con la presencia de rechazo crónico con estimación de riesgos. Se utilizaron estadísticas de tendencia central, tasas y proporciones para variables paramétricas, y comparación de medias de varia- 
Tabla 1: Resumen de estudios de laboratorio.

\begin{tabular}{|c|c|c|c|c|c|c|c|c|c|}
\hline & \multicolumn{3}{|c|}{ Hemoglobina ( $\mathrm{g} / \mathrm{dL})$} & \multicolumn{3}{|c|}{ Urea (mg/dL) } & \multicolumn{3}{|c|}{ Creatinina $(\mathrm{mg} / \mathrm{dL})$} \\
\hline & $\begin{array}{l}\text { Pretras- } \\
\text { plante }\end{array}$ & $\begin{array}{l}\text { Postras- } \\
\text { plante }\end{array}$ & Actual & $\begin{array}{l}\text { Pretras- } \\
\text { plante }\end{array}$ & $\begin{array}{l}\text { Postras- } \\
\text { plante }\end{array}$ & Actual & $\begin{array}{l}\text { Pretras- } \\
\text { plante }\end{array}$ & $\begin{array}{l}\text { Postras- } \\
\text { plante }\end{array}$ & Actual \\
\hline $\mathrm{N}$ & 53.000 & 52.000 & 41.0000 & 53.000 & 56.000 & 42.0000 & 53.000 & 55.000 & 42.0000 \\
\hline Media & 11.858 & 9.546 & 12.5805 & 107.147 & 76.332 & 56.8810 & 8.774 & 2.180 & 1.9214 \\
\hline Mediana & 11.600 & 9.450 & 12.8000 & 99.000 & 71.000 & 43.7500 & 7.900 & 1.500 & 1.2000 \\
\hline Mínimo & 6.500 & 6.100 & 5.0000 & 36.000 & 25.000 & 19.0000 & 2.700 & 0.700 & 0.8000 \\
\hline Máximo & 15.300 & 13.100 & 16.2000 & 253.000 & 223.000 & 173.0000 & 21.100 & 17.600 & 13.2000 \\
\hline
\end{tabular}

bles no paramétricas con prueba t de Student intragrupal y ANOVA, con confiabilidad $\alpha$ de $95 \%$ para un nivel de significancia estadística de $p<0.05$. Se utilizó el paquete estadístico SPSS v25 para el análisis.

\section{RESULTADOS}

Se trasplantó un total de 58 pacientes en el periodo de estudio de 14 años de 2005 a 2018, de los cuales ocho
(13.8\%) se realizaron en el periodo 2005-2008, 12 (20.7\%) en 2009-2013, 31 (53.4\%) en 2014-2017 y siete (12.1\%) en 2018. De ellos, 42 (72.4\%) fueron hombres y el promedio de edad fue de 44.2 años, con una mediana de 37.5 años, un mínimo de 14 y un máximo de 80 años. El tiempo promedio desde el trasplante fue de 4.96 años, con una mediana de 4.00 años y un rango de 0 a 14 .

$\mathrm{Al}$ agruparse por conjunto de edad con punto de corte de 10 años, 5.2\% $(n=3)$ tenían menos de 20 años, 46.6\%

\section{Tabla 2: Análisis de variables continuas de acuerdo con origen del riñón trasplantado.}

\begin{tabular}{|c|c|c|c|}
\hline & \multicolumn{3}{|c|}{ Media } \\
\hline & Vivo relacionado & Vivo no relacionado & Cadáver \\
\hline Edad & $42 a$ & $36 a$ & $49_{a}$ \\
\hline Tiempo de DX con ERC & 11 & $9_{a}$ & $9_{a}$ \\
\hline Tiempo desde trasplante (años) & $5 a$ & $3 a$ & $5 a$ \\
\hline Tiempo isquemia/ perfusión (min) & $64 a$ & $66_{a}$ & $454_{b}$ \\
\hline Hemoglobina pretrasplante (g/dL) & $11.3_{\mathrm{a}}$ & $11.7_{\mathrm{a}}$ & $12.3_{a}$ \\
\hline Urea pretrasplante (mg/dL) & $101.9 \mathrm{a}$ & $112.2_{a}$ & $114.6_{a}$ \\
\hline Creatinina pretrasplante (mg/dL) & $8.2 \mathrm{a}$ & $9.6 \mathrm{a}$ & $9.5_{a}$ \\
\hline Hemoglobina postrasplante (g/dL) & $9.6_{a, b}$ & $10.7_{\mathrm{a}}$ & $9.1_{b}$ \\
\hline Urea postrasplante (mg/dL) & $70.6_{a}$ & $54.0_{a}$ & $84.8_{a}$ \\
\hline Creatinina postrasplante (mg/dL) & $1.9_{a}$ & $1.1_{\mathrm{a}}$ & $2.7_{\mathrm{a}}$ \\
\hline Hemoglobina actual (g/dL) & $12.43_{a}$ & $13.17 \mathrm{a}$ & $12.21_{a}$ \\
\hline Urea actual (mg/dL) & $49.67 \mathrm{a}$ & $54.53_{a}$ & $66.18_{a}$ \\
\hline Creatinina actual (mg/dL) & $1.41_{\mathrm{a}}$ & $3.03 \mathrm{a}$ & $2.13_{a}$ \\
\hline
\end{tabular}


$(n=27)$ entre 20 y 39 años, $20.7 \%(n=12)$ entre 40 y 59 años y $27.6 \%(n=16)$ entre 60 y 80 años. La comparación entre género encontró una media de edad menor en mujeres (40 años) que en hombres (46 años), y en la comparación entre grupos de edad y género los porcentajes fueron similares, excepto en el grupo de 60-80 años (18.8\% en mujeres vs. $31 \%$ en hombres). El promedio de tiempo con diagnóstico de enfermedad renal crónica (ERC) fue de 10.2 años, (rango 3-21), y la gran mayoría (91.4\%) utilizaron hemodiálisis como terapia de sustitución renal, solamente un paciente diálisis peritoneal (1.7\%), y cuatro pacientes $(6.9 \%)$ no utilizaron terapia de sustitución renal (TSR) previo al trasplante. Las principales comorbilidades encontradas fueron hipertensión arterial sistémica (HAS)
(36.2\%), diabetes mellitus tipo 2 (DM2) (19\%), hipoplasia renal $(8.6 \%)$, epilepsia (5.2\%) y poliquistosis renal (3.4\%).

El estado bioquímico de los pacientes previo al trasplante, al egreso de la hospitalización del trasplante y los más actuales en el último año se resumen en la Tabla 1. Cabe destacar que el promedio de $\mathrm{Hb}$ previo fue de 11.8 $\mathrm{g} / \mathrm{dl}$, de urea de $107.1 \mathrm{mg} / \mathrm{dL}$ y de creatinina de $8.7 \mathrm{mg} /$ dL previo al trasplante.

En cuanto al origen del riñón, 43.4\% correspondieron a procedencia de donador vivo relacionado, con igual cifra a donador cadavérico, y $13.2 \%$ a donador vivo no relacionado.

El tiempo de isquemia-reperfusión, de acuerdo con lo esperado, fue más prolongado en riñón de donador

Tabla 3: Análisis de variables dicotómicas de acuerdo con origen del riñón trasplantado.

\begin{tabular}{|c|c|c|c|c|c|c|c|}
\hline & & \multicolumn{2}{|c|}{ Vivo relacionado } & \multicolumn{2}{|c|}{ Vivo no relacionado } & \multicolumn{2}{|c|}{ Cadáver } \\
\hline & & Recuento & $\begin{array}{l}\% \text { de } \mathrm{N} \\
\text { columnas }\end{array}$ & Recuento & $\begin{array}{l}\% \text { de } \mathrm{N} \\
\text { columnas }\end{array}$ & Recuento & $\begin{array}{l}\% \text { de } \mathrm{N} \\
\text { columnas }\end{array}$ \\
\hline \multirow[t]{2}{*}{ Género } & Femenino & $5 a$ & 21.7 & $3 a$ & 42.9 & $6_{a}$ & 26.1 \\
\hline & Masculino & $18_{a}^{a}$ & 78.3 & $4_{a}^{d}$ & 57.1 & $17_{\mathrm{a}}^{\mathrm{a}}$ & 73.9 \\
\hline \multirow{4}{*}{$\begin{array}{l}\text { Grupos edad } \\
\text { (años) }\end{array}$} & Menos de 20 & $2 a$ & 8.7 & $1_{\mathrm{a}}^{a}$ & 14.3 & $0^{1}$ & 0.0 \\
\hline & $20-39$ & $10_{a}^{a}$ & 43.5 & $4 a$ & 57.1 & $10_{a}$ & 43.5 \\
\hline & $40-59$ & $7_{\mathrm{a}}$ & 30.4 & $1_{\mathrm{a}}$ & 14.3 & $4_{a}$ & 17.4 \\
\hline & $60-80$ & $4_{a}^{d}$ & 17.4 & $1_{a}^{a}$ & 14.3 & $9_{a}^{a}$ & 39.1 \\
\hline \multirow{3}{*}{$\begin{array}{l}\text { Tiempo en TSR } \\
\text { (años) }\end{array}$} & $<1$ & $8 \mathrm{a}$ & 80.0 & $2 a, b$ & 50.0 & $5_{b}$ & 33.3 \\
\hline & $1-3$ & $2 a$ & 20.0 & $2 a$ & 50.0 & $8 a$ & 53.3 \\
\hline & $4-7$ & $0^{1}$ & 0.0 & $0^{1}$ & 0.0 & $2 a$ & 13.3 \\
\hline \multirow{4}{*}{$\begin{array}{l}\text { Periodo de } \\
\text { trasplante }\end{array}$} & 2018 & $4 a$ & 17.4 & $1_{a}$ & 14.3 & $2_{a}^{d}$ & 8.7 \\
\hline & 2014-2017 & $11_{\mathrm{a}}^{a}$ & 47.8 & $5_{a}^{a}$ & 71.4 & $13_{a}^{a}$ & 56.5 \\
\hline & $2009-2013$ & $5_{a}^{d}$ & 21.7 & $1_{a}^{d}$ & 14.3 & $6_{a}^{a}$ & 26.1 \\
\hline & $2005-2008$ & $3_{a}^{a}$ & 13.0 & $0^{a}$ & 0.0 & $2_{a}^{a}$ & 8.7 \\
\hline \multirow[t]{2}{*}{$\begin{array}{l}\text { Inducción de in- } \\
\text { munosupresión }\end{array}$} & $\begin{array}{l}\text { Metilprednisolona } \\
+ \text { basiliximab }\end{array}$ & $20_{a}^{a}$ & 90.9 & $7^{1}$ & 100.0 & $22_{a}^{d}$ & 95.7 \\
\hline & $\begin{array}{l}\text { Metilprednisolona } \\
+ \text { timoglobulina }\end{array}$ & $2 a$ & 9.1 & $0^{1}$ & 0.0 & $1_{\mathrm{a}}$ & 4.3 \\
\hline \multirow[t]{2}{*}{ Supervivencia } & Vivo & $16 \mathrm{a}$ & 94.1 & $6^{1}$ & 100.0 & $15_{\mathrm{a}}$ & 88.2 \\
\hline & Finado & $1_{\mathrm{a}}^{a}$ & 5.9 & $0^{1}$ & 0.0 & $2_{a}^{a}$ & 11.8 \\
\hline \multirow{2}{*}{$\begin{array}{l}\text { Necesidad TSR } \\
\text { postrasplante }\end{array}$} & No & $16^{1}$ & 100.0 & $5_{a}$ & 83.3 & $12_{a}^{a}$ & 80.0 \\
\hline & Sí & $0^{1}$ & 0.0 & $1_{a}^{a}$ & 16.7 & $3_{a}^{a}$ & 20.0 \\
\hline \multirow[t]{2}{*}{ Rechazo } & No & $22 a_{a}$ & 95.7 & $6_{a, b}$ & 85.7 & $17_{b}$ & 73.9 \\
\hline & Sí & $1_{a}^{a}$ & 4.3 & $1_{a, b}^{a, b}$ & 14.3 & $6 \mathrm{~b}$ & 26.1 \\
\hline Categoría & G1-G3 & $15_{a}$ & 93.8 & $5_{a}$ & 83.3 & $11_{a}$ & 84.6 \\
\hline KDIGO actual & G4-G5 & $1_{a}^{a}$ & 6.3 & $1_{a}^{a}$ & 16.7 & $2_{a}^{a}$ & 15.4 \\
\hline
\end{tabular}


Tabla 4: Análisis de variables continuas de acuerdo con rechazo crónico del injerto.

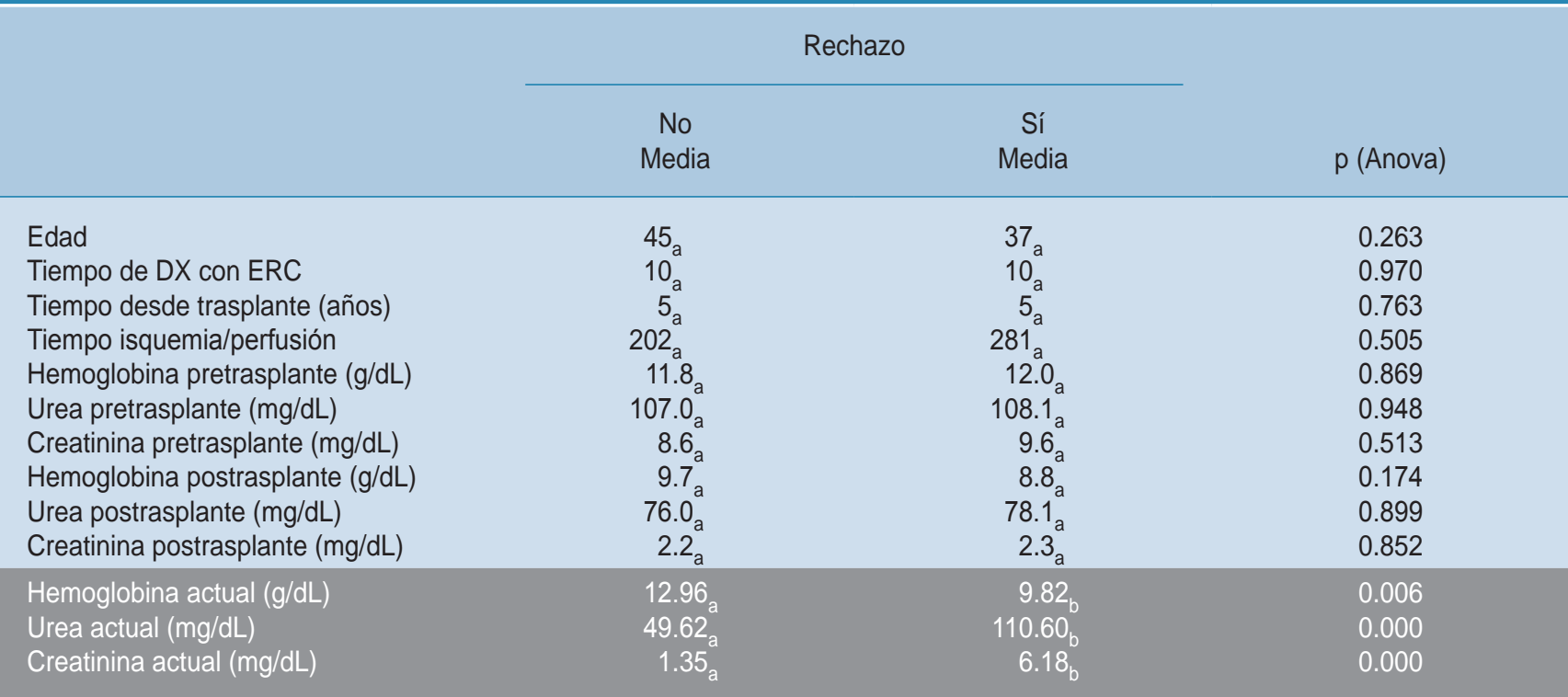

Nota: los valores de la misma fila y subtabla que no comparten el mismo subíndice son significativamente diferentes en $p<0.05$ en la prueba bilateral de igualdad para medias de columna.

cadavérico (454 vs. 66 minutos en riñón de donador vivo no relacionado y 64 minutos en riñón de donador vivo relacionado, $p<0.05)$. La hemoglobina postrasplante fue mayor en pacientes con riñón de donador vivo no relacionado (10.7 g/dL), seguidos de aquéllos con riñón de donador vivo relacionado $(9.6 \mathrm{~g} / \mathrm{dL})$ y donador cadavérico $(9.1 \mathrm{~g} / \mathrm{dL})(\mathrm{p}<0.05)$ (Tabla 2).

Se encontró diferencia significativa de acuerdo con el origen del riñón trasplantado en los pacientes en TSR con duración menor de un año (80\% en el grupo de donador vivo relacionado vs. $50 \%$ en el de vivo no relacionado y $33.3 \%$ en el de donador cadavérico, $p<0.05)$. De igual forma el rechazo del injerto se presentó con mayor frecuencia en el grupo de donador cadavérico (26.1\%) comparado con el de donador vivo no relacionado (14.3\%) y el de vivo relacionado $(4.3 \%)(p<0.05)$. Aunque no alcanzaron significancia estadística, la necesidad de TSR postrasplante $(0$ vs. $16.7 \%$ vs. $20 \%$ ), el porcentaje de pacientes con función renal en la Kidney Disease Improving Global Outcomes (KDIGO) G1-G3 (93.8 vs. $83.3 \%$ vs. $84.6 \%$ ) y la supervivencia (94.1 vs. $100 \%$ vs. $88.2 \%$ ), al comparar los grupos de vivo relacionado, vivo no relacionado y cadavérico, respectivamente, también mostraron una tendencia a favor del primer grupo (Tabla 3).

El porcentaje general de rechazo crónico fue de 13.8\%. Las variables con diferencia significativa al comparar los grupos sin rechazo vs. rechazo incluyeron las cifras más actuales de hemoglobina (12.9 vs. $9.8 \mathrm{~g} / \mathrm{dL})$, urea (49.6 vs. $110.6 \mathrm{mg} / \mathrm{dL}$ ) y creatinina (1.35 vs. $6.18 \mathrm{mg} / \mathrm{dL})$, respectivamente $(p<0.05 \%)$. Llama la atención que en las variables dicotómicas nominales y ordinales no se encontró significancia estadística en la comparación entre grupos de rechazo y no rechazo, excepto en el periodo de trasplante, siendo más prevalente el rechazo durante el periodo 20092013 (50 vs. 16\%) (Tablas 4 y 5).

El análisis de estimación de riesgos de rechazo mostró solamente significancia estadística para la necesidad de TSR postrasplante (RR 37.0, IC 95\% de 5.35-255.7).

De acuerdo con la clasificación KDIGO, 87.2\% de los pacientes se encuentran actualmente en G1-3 y $12.8 \%$ en KDIGO 4-5 (7.7\% en G1, 43.6\% en G2, 23.1\% en G3A, $12.8 \%$ en G3B, $7.7 \%$ en G4 y $5.1 \%$ en G5). Por grupos de edad, $100 \%$ de pacientes trasplantados menores de 20 años tienen KDIGO G1-G3, porcentaje que disminuye a 85.7 en aquéllos de $20-39$ años, a $85.7 \%$ de 40 a 59 años y $90 \%$ de $60-80$ años. El promedio de la tasa de filtrado glomerular (TFG) estimado por la fórmula de la Modification of Diet in Renal Disease (MDRD) fue de $55.7 \mathrm{~mL} / \mathrm{min} / 1.73$ $\mathrm{m}^{2}$ (mediana de 57).

\section{DISCUSIÓN}

El objetivo del presente estudio fue documentar la experiencia en trasplante renal en pacientes atendidos en el 
ámbito de la medicina privada por CENREN y el Hospital Ángeles del Carmen.

De los 58 trasplantes realizados, la mayoría fue en hombres $(72 \%)$, con promedio de edad de 44 años, y casi la mitad de ellos (47\%) entre 20 y 39 años de edad. Tenían un promedio de tiempo de 10 años con ERC, y la gran mayoría (91\%) con terapia sustitutiva renal con hemodiálisis. La mayoría (80\%) de los pacientes que recibieron trasplante de donador vivo relacionado estuvieron menos de un año en terapia sustitutiva renal previo al trasplante, en comparación con la mitad de los que recibieron trasplante de donador vivo no relacionado $(50 \%)$, y sólo una tercera parte $(33.3 \%)$ de los de donador cadavérico $(p<0.05)$. $55 \%$ de los pacientes padecían hipertensión arterial sisté- mica o diabetes mellitus tipo 2, y llegaron al trasplante con un promedio de hemoglobina de $11.8 \mathrm{~g} / \mathrm{dL}$, un promedio de urea de $107 \mathrm{mg} / \mathrm{dL}$ y un promedio de creatinina de $8.8 \mathrm{mg} / \mathrm{dL}$. 56.6\% correspondieron a trasplante de donador vivo y $43.4 \%$ de donador cadavérico. La distribución completa correspondió a $43.4 \%$ de donador vivo relacionado, $13.2 \%$ de donador vivo no relacionado y $43.4 \%$ de donador cadavérico. Egresaron de la hospitalización del trasplante con un promedio de $9.5 \mathrm{~g} / \mathrm{dL}$ de hemoglobina, de $76 \mathrm{mg} / \mathrm{dL}$ de urea y de $2.2 \mathrm{mg} / \mathrm{dL}$ de creatinina. Los receptores de riñón de donador cadavérico egresaron con cifras de hemoglobina más bajas que los de donador vivo relacionado y vivo no relacionado (9.1 vs. 9.6 y $10.7 \mathrm{~g} /$ $d L$, respectivamente $(p<0.05)$. Tras un promedio de casi

Tabla 5: Análisis de variables dicotómicas de acuerdo con rechazo crónico del injerto.

\begin{tabular}{|c|c|c|c|c|c|}
\hline & & \multicolumn{4}{|c|}{ Rechazo } \\
\hline & & \multicolumn{2}{|c|}{ No } & \multicolumn{2}{|c|}{ Sí } \\
\hline & & Recuento & $\begin{array}{l}\% \text { de } n \\
\text { columnas }\end{array}$ & Recuento & $\begin{array}{c}\% \text { de } n \\
\text { columnas }\end{array}$ \\
\hline \multirow[t]{2}{*}{ Género } & Femenino & $13_{a}$ & 26.0 & $3 a$ & 37.5 \\
\hline & Masculino & $37_{a}^{a}$ & 74.0 & $5_{a}^{a}$ & 62.5 \\
\hline \multirow[t]{4}{*}{ Grupos edad (años) } & Menos de 20 & $2_{a}^{a}$ & 4.0 & $1_{a}^{d}$ & 12.5 \\
\hline & $20-39$ & $23_{a}^{a}$ & 46.0 & $4_{a}^{a}$ & 50.0 \\
\hline & $40-59$ & $10_{a}^{d}$ & 20.0 & $2_{a}^{a}$ & 25.0 \\
\hline & $60-80$ & $15_{a}^{a}$ & 30.0 & $1_{a}^{a}$ & 12.5 \\
\hline \multirow[t]{3}{*}{ Tiempo en TSR (años) } & Menos 1 & $14_{a}^{a}$ & 50.0 & $2_{a}^{a}$ & 40.0 \\
\hline & $1-3$ & $13_{a}^{d}$ & 46.4 & $2_{a}^{a}$ & 40.0 \\
\hline & $4-7$ & $1_{a}^{d}$ & 3.6 & $1_{a}^{a}$ & 20.0 \\
\hline \multirow[t]{4}{*}{ Periodo de trasplante } & 2018 & $7_{a}^{d}$ & 14.0 & $0^{1}$ & 0.0 \\
\hline & $2014-2017$ & $27_{\mathrm{a}}^{a}$ & 54.0 & $4 a$ & 50.0 \\
\hline & $2009-2013$ & $8 \mathrm{a}$ & 16.0 & $4_{b}$ & 50.0 \\
\hline & $2005-2008$ & $8 a$ & 16.0 & 01 & 0.0 \\
\hline \multirow[t]{2}{*}{$\begin{array}{l}\text { Inducción de in- } \\
\text { munosupresión }\end{array}$} & $\begin{array}{l}\text { Metilprednisolona + } \\
\text { basiliximab }\end{array}$ & $46_{a}^{a}$ & 93.9 & $8^{1}$ & 100.0 \\
\hline & $\begin{array}{l}\text { Metilprednisolona + } \\
\text { timoglobulina }\end{array}$ & $3 a$ & 6.1 & 01 & 0.0 \\
\hline \multirow[t]{2}{*}{ Supervivencia } & Vivo & $36 a$ & 94.7 & 5 & 83.3 \\
\hline & Finado & $2_{a}^{a}$ & 5.3 & $1_{a}^{a}$ & 16.7 \\
\hline Necesidad de TSR & No & $36^{1}$ & 100.0 & $1_{a}^{a}$ & 20.0 \\
\hline postrasplante & Sí & $0^{1}$ & 0.0 & $4_{a}^{a}$ & 80.0 \\
\hline \multirow[t]{2}{*}{ Categoría KDIGO actual } & G1-G3 & $34 a$ & 94.4 & $0^{a}$ & 0.0 \\
\hline & G4-G5 & $2_{a}^{a}$ & 5.6 & 31 & 100.0 \\
\hline
\end{tabular}


cinco años de seguimiento tienen cifras actuales promedio de hemoglobina de $12.5 \mathrm{~g} / \mathrm{dL}$, de urea de $56 \mathrm{mg} / \mathrm{dL}$ y de creatinina de $1.9 \mathrm{mg} / \mathrm{dL}$.

La gran mayoría (> 90\%) recibió inducción de inmunosupresión con metilprednisolona y basiliximab en comparación con metilprednisolona y timoglobulina $(<10 \%)$, de manera homogénea en los tres grupos de receptores. La sobrevida a cinco años promedio del trasplante es mayor de $88 \% \sin$ diferencias estadísticas significativas, aunque es menor en receptores de donador cadavérico (88\%) comparado con donador vivo relacionado (94\%) y donador vivo no relacionado (100\%). El rechazo crónico se ha presentado en $13.8 \%$ de los 58 pacientes trasplantados. De ellos, $26.1 \%$ corresponde a receptores de donador cadavérico, en comparación con $14.3 \%$ en receptores de donador vivo no relacionado y $4.3 \%$ de vivo relacionado $(p<0.05)$. Siguiendo dicha tendencia, aunque sin alcanzar la significancia estadística, 20\% de los receptores de donador cadavérico han necesitado terapia de sustitución renal posterior al trasplante, mientras que sólo $16.7 \%$ de los de donador vivo no relacionado lo han requerido, y ninguno de los de donador vivo relacionado. La necesidad de TSR postrasplante fue un factor de riesgo muy significativo de presentar rechazo (RR 37.0, IC 95\% de 5.35-255.7, $p<0.05)$. De igual manera, es claramente mayor el porcentaje de pacientes que ha mantenido una función renal en grado KDIGO 1-3 (93.8\% en receptores de donador vivo relacionado, en comparación con 83.3 y $84.6 \%$ en receptores de donador vivo no relacionado y cadavérico, respectivamente). El promedio de TFGe por MDRD a cinco años fue de $55.7 \mathrm{~mL} / \mathrm{min} / 1.73 \mathrm{~m}^{2}$. Las cifras promedio de hemoglobina actual son mayores en pacientes que no tuvieron rechazo (12.96 vs. $9.82 \mathrm{~g} / \mathrm{dL}, \mathrm{p}=0.006$ ), y los niveles de azoados claramente menores (urea de 49.6 vs. $110.6 \mathrm{mg} / \mathrm{dL}, \mathrm{p}<0.001$; creatinina de 1.35 vs. 6.18, $\mathrm{p}<0.001$ ).

Al hacer una comparación con otras series de trasplante reportadas en la literatura, tanto de primer mundo como en países en vías de desarrollo, es difícil lograr un análisis equitativo, ya que las series muestran mucha heterogeneidad en el número de pacientes, incluidos el origen del riñón trasplantado, el periodo en que se hizo el estudio y el tiempo que abarcó.

A excepción del estudio de Portugal, ${ }^{11}$ los realizados en países desarrollados (EUA y España) ${ }^{12,13}$ se llevaron a cabo antes de 2005. Sólo los estudios reportados de países en vías de desarrollo (Nigeria, Egipto), 7,14 y los estudios realizados en México por Morales-Buenrostro y colaboradores $(2005-2018)^{9}$ y por Contreras y su equipo $(2011-2017)^{10}$ compartieron de manera parcial el periodo que abarcó el presente estudio.

Con reserva de la diferencia de tiempo en que fueron realizados, no se reportan trasplantes de donante cadavéri- co efectuados en otros países en vías de desarrollo estudiados. La experiencia en México demuestra un aumento paulatino desde lo reportado por Monteón y colegas (10\%), ${ }^{5}$ Morales-Buenrostro y colaboradores $(19.3 \%)^{9}$ y Contreras y su equipo $(37.9 \%)^{10}$ hasta nuestra experiencia (43.4\%).

Nuestro estudio comparte mayor similitud de características con países desarrollados que con países en vías de desarrollo, específicamente en mediana o promedio de edad, porcentaje de receptores entre 18 y 54 años, porcentaje de pacientes en hemodiálisis previo al trasplante, porcentaje de pacientes en TSR $<$ seis años antes del trasplante y supervivencia a cinco años. Sólo en proporción de hombres, ésta es similar a la reportada por países en vías de desarrollo (Nigeria, Egipto) ${ }^{7,14}$ y otras características guardan similitud con países de ambos estados de desarrollo como la proporción de pacientes en hemodiálisis (parecida a la descrita por Nigeria y Portugal) 7,11 y la DM como causa de ERC (parecida a Nigeria y EUA). ${ }^{7,13}$

Cabe resaltar que el tiempo de isquemia fría en trasplante de donante cadavérico es significativamente menor en nuestra serie (7.5 horas) en comparación con el reportado en países desarrollados (17.6 horas en Portugal, 21 horas en EUA y 19 horas en España). ${ }^{11,13}$

En el tema de inducción de la inmunosupresión, guías recientes recomiendan que se utilice un antagonista del receptor de IL-2 (basiliximab) como agente de primera línea y se reserve el uso de agentes depletores de linfocitos para receptores con alto riesgo inmunológico. ${ }^{15}$ En nuestra serie el uso de basiliximab fue $>90 \%$, y no se reporta en las demás, excepto las series mexicanas, pero con porcentajes mucho menores (5.7 y $12 \%) .5$

La tasa de rechazo crónico en nuestra serie (13.8\%) se encuentra en punto intermedio entre las cifras registradas en EUA $(3 \%)^{12}$ y las de Egipto (19-23\%). ${ }^{14}$ Es comparable a lo reportado en la década de 1980 en EUA (15\%). Cabe resaltar que en Egipto es más elevada tanto en trasplante de donante vivo relacionado (23.6 vs. $4.3 \%$ ) como no relacionado (19.5 vs. $14.3 \%$ ).

La necesidad de TSR postrasplante en trasplante de donante cadavérico en nuestra serie es menor que en Portugal (20 vs. 38.1\%), aunque mayor en trasplante de donante vivo (16.7\% en DVNR vs. $5 \%$ ). ${ }^{11}$ Se debe tomar en cuenta que en trasplante de donante vivo relacionado fue de $0 \%$.

La supervivencia general a cinco años fue de 93\%; sin embargo, a diferencia de la mayoría de las demás series, la nuestra incluyó receptores de donantes cadavéricos (supervivencia de $88 \%$ ) vivos no relacionados (supervivencia de $100 \%$ ) y relacionados (95\%). La única serie que reporta supervivencia a cinco años de donantes cadavéricos es la de Monteón y colaboradores, la cual es 10\% menor (78 vs. $88 \%) .{ }^{5}$ En receptores de donantes vivos la supervivencia 
Tabla 6: Comparativo de diferentes series publicadas sobre trasplante renal.

\begin{tabular}{|c|c|c|c|c|c|c|c|c|c|c|c|c|}
\hline & $\begin{array}{c}\text { EUA } \\
\left(\text { Matas) }^{12}\right.\end{array}$ & $\begin{array}{c}\text { EUA } \\
(\text { Ojo })^{13}\end{array}$ & $\begin{array}{c}\text { España } \\
(\text { Ojo })^{13}\end{array}$ & $\begin{array}{l}\text { Por } \\
\text { (Guim }\end{array}$ & $\begin{array}{l}\text { ugal } \\
\text { raes })^{11}\end{array}$ & $\begin{array}{r}\mathrm{Ec} \\
(\mathrm{Ma}\end{array}$ & $\begin{array}{l}\text { pto } \\
\text { er) }{ }^{14}\end{array}$ & $\begin{array}{c}\text { Nigeria } \\
\text { (Hugues) }^{7}\end{array}$ & $\begin{array}{c}\text { México } \\
\text { CMNO } \\
\text { (Monteón) }^{5}\end{array}$ & $\begin{array}{c}\text { México } \\
\text { INCMNSZ } \\
\text { (Morales) }^{9}\end{array}$ & $\begin{array}{l}\text { México } \\
\text { HAP } \\
\text { (Contre- } \\
\text { ras) })^{10}\end{array}$ & $\begin{array}{l}\text { México } \\
\text { HAC } \\
\text { (García- } \\
\text { Otero) }\end{array}$ \\
\hline $\mathrm{n}$ & 2,540 & 9,609 & 3,808 & & & & & 47 & 1,356 & 923 & 29 & 58 \\
\hline $\begin{array}{ll}\text { Tipo } & \text { Vivo } \\
\text { de do- } & \\
\text { nante } & \end{array}$ & $\begin{array}{l}100 \% \\
\text { (DVR) }\end{array}$ & & & & & $\begin{array}{r}100 \% \\
\text { DVR } \\
\text { DV }\end{array}$ & $\begin{array}{l}(84 \% \\
16 \% \\
\text { JR) }\end{array}$ & $\begin{array}{c}100 \% \\
(25.5 \% \\
\text { DVR, } \\
74 \% \\
\text { DVNR) }\end{array}$ & $\begin{array}{l}90 \%(82 \% \\
\text { DVR, } 8 \% \\
\text { DVNR) }\end{array}$ & $\begin{array}{c}80.7 \% \\
(74.5 \% \\
\text { DVR, } \\
6.2 \% \\
\text { DVNR) }\end{array}$ & $62.1 \%$ & $\begin{array}{c}56.6 \% \\
(43.4 \% \\
\text { DVR, } \\
13.2 \% \\
\text { DVNR) }\end{array}$ \\
\hline $\begin{array}{l}\text { Muerte en- } \\
\text { cefálica (\%) }\end{array}$ & & 100 & 100 & & & & & & 10 & 19.3 & 37.9 & 43.4 \\
\hline Duración (años) & 35 & 12 & 12 & $23 n$ & eses & & & 4 & 27 & 40 & 6 & 14 \\
\hline Periodo & $\begin{array}{l}1963- \\
1998\end{array}$ & $\begin{array}{c}1990 \\
1994,1998 \\
\text { y } 2002\end{array}$ & $\begin{array}{c}1990 \\
1994,1998 \\
\text { y } 2002\end{array}$ & 2012 & 2013 & $197 €$ & 2013 & $\begin{array}{l}2010- \\
2014\end{array}$ & $1976-2003$ & $\begin{array}{l}1967- \\
2009\end{array}$ & $2011-2017$ & $2005-2018$ \\
\hline $\begin{array}{l}\text { Edad (mediana o } \\
\text { media) }\end{array}$ & $\begin{array}{c}\text { 1963-89: } \\
30,1990- \\
98: 37\end{array}$ & 48 & 49 & 49.9 & 37.2 & 36.2 & 31.4 & 45.4 & 31 & 33.9 & 51 & 44 \\
\hline Hombres (\%) & $\begin{array}{c}1963- \\
89: 61 \% \\
1990-98: \\
64 \%\end{array}$ & 63.1 & 61.8 & 62 & 24 & 74.9 & 72.4 & 76 & 64 & 58.4 & 79.3 & 72 \\
\hline $\begin{array}{l}\text { Receptores }<35 \\
\text { años (\%) }\end{array}$ & & 17 & 21 & & & & & & & & & 47 \\
\hline $\begin{array}{l}\text { Receptores > } 70 \\
\text { años (\%) }\end{array}$ & & 3.50 & 1.70 & & & & & & & & & 15.5 \\
\hline $\begin{array}{l}\text { Hemodiálisis como } \\
\text { TSR (\%) }\end{array}$ & & & & 94 & 19 & & & 98 & 21 & & 75.9 & 91 \\
\hline TSR < 6 años $(\%)$ & & 95.0 & 90.0 & & & & & & & & & 93.9 \\
\hline $\begin{array}{l}\text { DM como causa } \\
\text { primaria de ERCT } \\
(\%)\end{array}$ & $\begin{array}{c}\text { 1963- } \\
\text { 89: } 1 \% \\
\text { 1990-98: } \\
5 \%\end{array}$ & 24.0 & 6.0 & 11 & 1 & & & 15 & 3 & 5.8 & 24.1 & 19 \\
\hline $\begin{array}{l}\text { HAS como } \\
\text { causa primaria } \\
\text { de ERCT }(\%)\end{array}$ & 2 & 13.0 & 7.0 & 5 & 1 & 57.6 & 57.1 & 24 & 1.5 & & 37.9 & 36.2 \\
\hline
\end{tabular}


Continuación de la Tabla 6: Comparativo de diferentes series publicadas sobre trasplante renal.

\begin{tabular}{|c|c|c|c|c|c|c|c|c|c|c|c|c|}
\hline \multirow{2}{*}{$\begin{array}{l} \\
\text { Inducción de inmu- } \\
\text { nosupresión con } \\
\text { timoglobulina (\%) }\end{array}$} & \multirow[t]{2}{*}{$\begin{array}{c}\text { EUA } \\
(\text { Matas) })^{12}\end{array}$} & \multirow[t]{2}{*}{$\begin{array}{l}\text { EUA } \\
(\mathrm{Ojo})^{13}\end{array}$} & \multirow[t]{2}{*}{$\begin{array}{l}\text { España } \\
(\text { Ojo })^{13}\end{array}$} & \multicolumn{2}{|c|}{$\begin{array}{c}\text { Portugal } \\
\text { (Guimaraes) }^{11}\end{array}$} & \multicolumn{2}{|c|}{$\begin{array}{c}\text { Egipto } \\
\text { (Matter) }^{14}\end{array}$} & $\begin{array}{c}\text { Nigeria } \\
\text { (Hugues) }^{7}\end{array}$ & $\begin{array}{c}\text { México } \\
\text { CMNO } \\
\text { (Monteón) }^{5}\end{array}$ & \multirow{2}{*}{$\begin{array}{c}\begin{array}{c}\text { México } \\
\text { INCMNSZ } \\
\text { (Morales) }\end{array} \\
1.7\end{array}$} & \multirow[t]{2}{*}{$\begin{array}{c}\text { México } \\
\text { HAP } \\
\text { (Contre- } \\
\text { ras) })^{10}\end{array}$} & \multirow{2}{*}{$\begin{array}{c}\text { México } \\
\text { HAC } \\
\text { (García- } \\
\text { Otero) }\end{array}$} \\
\hline & & & & & & 6.9 & 16 & & & & & \\
\hline $\begin{array}{l}\text { Inducción de inmu- } \\
\text { nosupresión con } \\
\text { basiliximab (\%) }\end{array}$ & & & & & & & & & 12 & 5.7 & & 91 \\
\hline $\begin{array}{l}\text { Rechazo crónico } \\
(\%)\end{array}$ & $\begin{array}{c}\text { 1960: } \\
30 \% \\
\text { 1970: } \\
\text { 18\%, } \\
1980: \\
15 \% \\
1990: 3 \%\end{array}$ & & & & & 23.6 & 19.5 & & & & & $\begin{array}{c}13.8 \% \\
(26.1 \% \text { en } \\
\text { ME, } 14.3 \% \\
\text { en DVNR } \\
\text { y } 4.3 \% \text { en } \\
\text { DVR) }\end{array}$ \\
\hline $\begin{array}{l}\text { TSR postrasplante } \\
(\%)\end{array}$ & & & & 38.1 & 5 & & & & & & & $\begin{array}{c}9.8 \% \text { (20\% } \\
\text { de ME, } \\
16.7 \% \text { de } \\
\text { DVNR y } \\
\text { 0\% DVR) }\end{array}$ \\
\hline $\begin{array}{l}\text { Tasa de supervi- } \\
\text { vencia del paciente } \\
\text { a cinco años }\end{array}$ & $\begin{array}{c}93 \% \text { sin } \\
\text { TSR, } \\
95 \% \text { con } \\
\text { TSR }< \\
1 \text { año y } \\
81 \% \text { con } \\
\text { TSR > } 1 \\
\text { año }\end{array}$ & & & & & $95.1 \%$ & $88.8 \%$ & $\begin{array}{l}67.9 \%(4 \\
\text { años) }\end{array}$ & $\begin{array}{l}\text { DV } 81 \% \text {, } \\
\text { ME } 78 \%\end{array}$ & & & $\begin{array}{c}93 \%(88 \% \\
\text { ME, } 95 \% \\
\text { DVR y } \\
100 \% \\
\text { DVNR) }\end{array}$ \\
\hline $\begin{array}{l}\text { TFGe a los cinco } \\
\text { años (MDRD) }\end{array}$ & & 50.6 & 50.1 & & & & & & & & & $\begin{array}{c}55.7 \\
\text { (74.4\% con } \\
\text { TFGe > } \\
45 \mathrm{ml} / \mathrm{min}) \\
\text { (KDIGO } \\
\text { G1-3 93.8\% } \\
\text { en DVR, } \\
\text { 83.3\% en } \\
\text { DVNR y } \\
\text { 84.6\% en } \\
\text { DC) }\end{array}$ \\
\hline
\end{tabular}


es claramente mayor en nuestra serie (96\%) comparada con $81 \%$ en Pakistán y el estudio previo en México de Monteón y su equipo y $67.9 \%$ en Nigeria (este último reportado a cuatro años). 7,8 Si se hace la diferencia por donante vivo relacionado y no relacionado, la serie de EUA registró $90 \%$ de supervivencia a cinco años, mientras que la egipcia y la nuestra reporta $95 \% .{ }^{12,14}$ En receptores de donante vivo no relacionado la sobrevida es claramente mayor en nuestro estudio (100\%) comparada con la serie egipcia $(88 \%){ }^{14}$

La función renal a cinco años es mayor que la descrita en países desarrollados (TFGe por MDRD de 55.7 $\mathrm{mL} / \mathrm{min} / 1.73 \mathrm{~m}^{2}$ vs. 50.6 en EUA y 50.1 en España). ${ }^{13}$ Además, $74.4 \%$ de los receptores tuvieron TFGe a cinco años > $45 \mathrm{~mL} / \mathrm{min} / 1.73 \mathrm{~m}^{2}$, y $93.8 \%$ de receptores de donantes vivos relacionados mantenían una clasificación KDIGO G1-3, cifra que disminuyó a 83\% en DVNR y 85\% en DC (Tabla 6).

\section{CONCLUSIÓN}

Los resultados en pacientes con trasplante renal en la práctica privada en México son más parecidos a los reportados por países desarrollados, algunos incluso mejores que los reportados en la literatura, tales como el tiempo de isquemia fría en trasplante de donante cadavérico, el uso de basiliximab y la función renal a cinco años.

\section{REFERENCIAS}

1. Hatzinger $M$, Stastny $M$, Grützmacher P, Sohn M. The history of renal transplantation. Urologue A. 2016; 55 (10): 1353-1359.
2. Barker CF, Markmann JF. Historical overview of transplantation. Cold Spring Harb Perspect Med. 2013; 3: a014977.

3. Murray JE, Merrill JP, Harrison JH, Wilson RE, Dammin GJ. Prononged survival of human-kidney homografts by immunosuppressive drug therapy. N Eng J Med. 1963; 268: 1315-1323.

4. Cecka JM. Kidney transplantation in the United States. Clin Transp. 2008: 1-18.

5. Monteón F, Gómez B, Valdespino C, Chávez S, Sandoval M, Flores A et al. The kidney transplant experience at Hospital de Especialidades, Centro Médico Nacional de Occidente, IMSS, Guadalajara, México. Clin Transpl. 2003; 165-174.

6. Baptista-González HA. Trasplante renal, a propósito de los primeros casos. Rev Invest Med Sur Mex. 2011; 18 (1): 32-34.

7. Hugues U. Kidney transplant in Nigeria: a single centre experience. 2016. Pan Afr Med J. 2016; 25.

8. Hasan S, Naqvi A, Hussain Z, Hashmi A, Akhtar F, Hussain M et al. Renal transplantation in developing countries. Kidney Int Suppl. 2003; 63 (83): S96-100.

9. Morales-Buenrostro LE, Marino-Vázquez LA, Alberú J. Four decades of the kidney transplantation program at the Institute Nacional de Ciencias Médicas y Nutrición Salvador Subirán in Mexico City. Clin Transp. 2009; 153-164.

10. Contreras C, García A, Hernández C, Portela J. Experiencia perioperatoria en el trasplante renal en un Hospital Privado en México. Acta Médica Grupo Ángeles. 2019; 17 (2): 126-130.

11. Guimarães J, Araújo A, Santos F, Nunes C, Casal M. Living-donor and deceased-donor renal transplantation: differences in early outcome-a single-center experience. Transplant Proc. 2015; 47: 958-962.

12. Matas A, Payne W, Sutherland D, Humar A, Gruessner R, Kandaswamy $R$ et al. 2,500 living donor kidney transplants: a single-center experience. Ann Surg. 2001; 234 (2): 149-164.

13. Ojo A, Morales J, González-Molina M, Steffick D, Luan F, Merion R et al. Comparison of the long-term outcomes of kidney transplantation: USA versus Spain. Nephrol Dial Transplant. 2013; 28: 213-220.

14. Matter Y, Nagid A, Lotfy O, Alsayed A, Donia A, Redaie A. Impact of donor source on the outcome of live donor kidney transplantation: a single center experience. Nephrourol Mon. 2016; 8 (3): 1-8.

15. Hardinger K, Brennan D, Klein C. Selection of induction therapy in kidney transplantation. Transpl Int. 2012; 26: 662-672. 